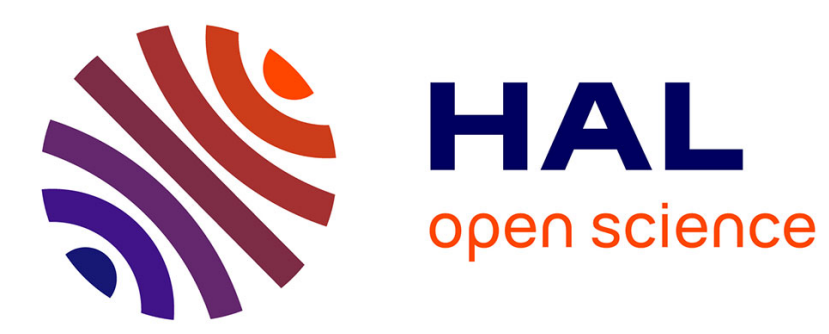

\title{
Theoretical study of graphene doping mechanism by iodine molecules
}

\author{
Damien Tristant, Pascal Puech, I.C. Gerber
}

\section{To cite this version:}

Damien Tristant, Pascal Puech, I.C. Gerber. Theoretical study of graphene doping mechanism by iodine molecules. Journal of Physical Chemistry C, 2015, 119 (21), pp.12071-12078. 10.1021/acs.jpcc.5b03246 . hal-01756984

\section{HAL Id: hal-01756984 \\ https://hal.science/hal-01756984}

Submitted on 30 Jan 2019

HAL is a multi-disciplinary open access archive for the deposit and dissemination of scientific research documents, whether they are published or not. The documents may come from teaching and research institutions in France or abroad, or from public or private research centers.
L'archive ouverte pluridisciplinaire HAL, est destinée au dépôt et à la diffusion de documents scientifiques de niveau recherche, publiés ou non, émanant des établissements d'enseignement et de recherche français ou étrangers, des laboratoires publics ou privés. 


\title{
Theoretical study of graphene doping mechanism by iodine molecules
}

\author{
Damien Tristant, ${ }^{\dagger, \ddagger}$ Pascal Puech, ${ }^{\ddagger}$ and lann C. Gerber ${ }^{*, \dagger}$ \\ Université de Toulouse; INSA, UPS, CNRS; \\ LPCNO, 135 avenue de Rangueil, F-31077 Toulouse, France, and CEMES, UPR 8011, \\ CNRS-Université de Toulouse, 29 rue Jeanne Marvig, BP 94347, 31055 Toulouse, France \\ E-mail: igerber@insa-toulouse.fr
}

\begin{abstract}
The adsorption of iodine atoms and molecules on graphene is studied in detail, using first-principles calculations that include non-local correlation effects by means of van der Waals density functional approach. Structural, energetic and electronic structure properties of these systems are reported. We demonstrate that graphene surface can be doped by atomic and molecular iodine. An upward shift of the Dirac point from the Fermi Level, with values of $0.45 \mathrm{eV}$ and $0.08 \mathrm{eV}$ are observed, for adsorbed atoms and adsorbed $\mathrm{I}_{2}$ respectively . It corresponds to graphene hole densities to be around $1.2 \times 10^{13} \mathrm{~cm}^{-2}$ to $3.9 \times 10^{11} \mathrm{~cm}^{-2}$. We also show that the iodine molecule does not dissociate in contact with pure graphene monolayer. Calculation of the surface free energy reveals that the orientation of the adsorbed iodine molecules crucially depends on its concentration and the system temperature. The corresponding phase diagram
\end{abstract}

\footnotetext{
${ }^{*}$ To whom correspondence should be addressed

†Université de Toulouse; INSA, UPS, CNRS;

LPCNO, 135 avenue de Rangueil, F-31077 Toulouse, France

${ }^{\ddagger}$ CEMES, UPR 8011, CNRS-Université de Toulouse, 29 rue Jeanne Marvig, BP 94347, 31055 Toulouse, France
} 
indicates that the in-plan orientation of molecules is more stable when the iodine concentration decreases for temperature above approximately $200 \mathrm{~K}$, when beyond $500 \mathrm{~K}$, iodine molecules are completely desorbed.

\section{Introduction}

Graphene layer is one of the most prominent nanoscale material currently studied. The confinement in two dimensions and the high symmetry of this two dimensional material lead to interesting electronic and transport properties. Indeed graphene is a zero-gap semiconductor with a linear dispersion of the conduction and valence bands at the Fermi level. Important efforts are made to tune these properties, among them the adsorption of halogen molecules on the surface of graphene mono-layer is a promising approach, due to the diversity of halogen element properties and the variety of structures formed.1

The adsorption of halogen atoms and molecules on graphene layer has been studied theoretically as well as experimentally to tune the electronic structure of graphene layer. ${ }^{2[10}$ Rudenko et al. ${ }^{[2]}$ were the first to study, at the density functional theory (DFT) level, the adsorption of diatomic halogen molecules on graphene using van der Waals vdW-DF functional, 11 that includes non-local correlation effects seamlessly, for geometry optimizations. Neglecting dispersion effects on the electronic structures, they conclude that iodine molecules withdraw electrons from graphene. More recently Luo et al., ${ }^{5}$ have studied the adsorption of halogen atoms on graphene using a semi-local functional only. Again they state that an iodine atom can accept 0.5 electron from the carboneous substrate. These results are in line with previous studies: 0.38 and 0.46 electron from Ref. [6] and Ref. 7 respectively. Besides, very recently a work combining experimental and theoretical determination, of the adsorption strength, and of the charge transfer for $\mathrm{Br}_{2}$ in gaz phase in interaction with graphene appears. $\frac{12}{12}$ The maximum charge transfer is obtained when the graphene is fully covered by $\mathrm{Br}_{2}$ molecules, however the corresponding hole densities extracted from DFT calculations remains one order of magnitude smaller than the experimental data. 
On the experimental side, Chu et al. $\frac{3}{3}$ show that upon adsorption a Raman shift of the G-peaks, around $7.1 \mathrm{~cm}^{-1}$, is observed indicating successful doping with iodine molecules. In fact if the Dirac cone is preserved, 13 the Raman G band shift due to p-doping corresponds to a hole areal density of $8 \times 10^{12} \mathrm{~cm}^{-2} .14$ Ghosh et al. ${ }^{[4}$ study the interaction of halogen molecules on graphene and they find that the decrease in the $\mathrm{I}_{2 \mathrm{D}} / \mathrm{I}_{\mathrm{G}}$ ratio is minimum for $\mathrm{I}_{2}$. It demonstrates that the magnitude of molecular charge-transfer between iodine and graphene is the lowest among the halogens. More recently solution-deposition of $\mathrm{I}_{2}$ adsorbed on graphene surface have been performed by D'Arsié et al. $[8$ Electrical measurements show that the dopants increase the graphene sheet conductivity by $19 \%$ around $300^{\circ} \mathrm{C}$, without deteriorating its lattice structure. In the same spirit, it has been shown that the conductivity of carbon nanotubes-based fibers doped by iodine can exceed the electrical conductivity of metals, $\frac{15}{15}$ but the origin of the enhancement remains unclear.

In this work, the adsorption of atomic and molecular iodine species at various concentrations on graphene layer is studied using fully self-consistently the non-local optB86b-vdW functional ${ }^{16}$ We present our investigations of the structural, energetic properties as well as the electronic structure of graphene monolayer decorated by iodine species. Computational details are described in Sec.II. Then our results of adsorbed iodine atoms and molecules are reported in Sec.III and Sec.IV respectively. We show that an electron transfer of $0.46 \mathrm{e}$ from the graphene layer to an iodine atom is obtained suggesting an efficient hole doping of graphene, by atomic iodine. Interestingly we prove that $\mathrm{I}_{2}$ molecules do not dissociate spontaneously on a pristine graphene monolayer, since molecular state is more stable that the dissociated one, at any coverage values. Simulation of the thermodynamic properties of adsorbed $I_{2}$ as a function of the coverage and the iodine chemical potential are also presented. This allows us to prove that a modification of the iodine concentration leads to a phase transition in the adsorption modes. We conclude, in the Sec.V, that the conductivity of graphene can be adjusted by changing the concentration of $\mathrm{I}_{2}$. The calculated electronic transfer suggests a p-doping of graphene, as reported in the literature. 


\section{Computational details}

For the carbon structures, we use a lattice constant of graphene equal to $a_{0}=2.46$ $\AA$, close to the experimentally obtained value $(2.459 \AA)$ for a graphite at low temperatures. ${ }^{17}$ We also add a vacuum region of $20 \AA$ to avoid interaction between periodic images in the $z$ direction. In order to determine the effects of the adsorbed impurity concentrations, several hexagonal cells containing from 2 to 200 carbon atoms in interaction with iodine atoms and molecules are considered. So we use $(1 \times 1)$ to $(10 \times 10)$ primitive cells of graphene, where $(1 \times 1)$ and $(10 \times 10)$ corresponds to $50 \%\left(\mathrm{C}_{2} \mathrm{I}\right.$ or $\left.\mathrm{C}_{2} \mathrm{I}_{2}\right)$ and $0.50 \%\left(\mathrm{C}_{200} \mathrm{I}\right.$ or $\left.\mathrm{C}_{200} \mathrm{I}_{2}\right)$ of iodine concentration respectively. In the following, we investigate three high symmetry adsorption sites of iodine atom on graphene, i.e the $B$ Bridge, $H$ hollow and $T$ Top sites, as illustrated in Fig, 1 . Besides we consider six in-plan (subscribed with $\|$ ) and three perpendicular (subscribed with $\perp$ ) configurations of adsorbed iodine molecule, see Fig 2. Isolated iodine molecule is simulated, in a

$36 \times 36 \times 36 \AA^{3}$ box, yielding an interatomic distance of $2.67 \AA$ at best, depending of the exchange-correlation functional choice, a value very close to the experimental one $(2.666$ $\AA) .18$ To validate our choice of xc-functional, we have assess several combinations of exchange and correlation functionals, against several geometric parameters. Among them we have studied a graphite structure containing 4 carbon atoms in its primitive cell with an interlayer distance of $3.35 \AA$ in good agreement with experimentally obtained value. 19

The structural and electronic properties of iodine-graphene systems are obtained using the Vienna ab initio simulation package (VASP). 20.23 Ions cores are modeled with projector augmented wave (PAW) pseudopotentials. 24 The $2 s$ and $2 p$ states of carbon, $5 s$ and $5 p$ states of iodine are treated explicitly in the valence. The plane-wave basis set cutoff energy is set to $400 \mathrm{eV}$ with a Gaussian smearing method ${ }^{25}$ of 0.005 $\mathrm{eV}$ width, in order to assure well converged total energy and force values. To include van der Waals interactions in our calculations, we use the vdW-DF scheme proposed by Dion et al.. $\stackrel{11}{ }$ It is designed to handle both short-range covalent bonds and long- 
range van der Waals interactions without inserting damping functions or empiricism. One of the first possibility is to combine the revPBE ${ }^{26}$ exchange energy, with the local-density approximation (LDA) correlation energy and the non-local correlation correction energy term. However others choices are possible such as vdW-DF2, optPBEvdW, optB88-vdW and optB86b-vdW. ${ }^{27}$ We also include LDA and PBE ${ }^{28}$ results for sake of comparison.

All the atoms are allowed to relax until the maximum of all forces acting on them became smaller than $0.01 \mathrm{eV} . \AA^{-1}$. The k-point sampling is always based on a $\Gamma$-centered grid for all types of calculations. We use, to optimize the structures, a single k-point for molecule iodine, and a $(19 \times 19 \times 1)$ grid for primitive cells of graphite and graphene up to a $(5 \times 5 \times 1)$ grid for the largest graphene supercell. For the density of states (DOS) calculations, the tetrahedron integration method with Blöchl corrections ${ }^{29}$ is used, with a $(23 \times 23 \times 1)$ grid for the primitive cell of graphene and a $(9 \times 9 \times 1)$ grid for the $(10 \times 10)$ graphene supercell. Similar grids are used to determine charge transfer and band structures for graphene systems. Bader charge analysis is carried out using Henkelman's group program. $30+32$

We can compare the energy stability of all structures via the adsorption energy $E_{\mathrm{ad}}^{(n, m)}$ of doped graphene calculated from the following equation:

$$
E_{\mathrm{ad}}^{(n, m)}=E_{\mathrm{I}_{n} @ \text { graphene }}-\frac{n}{m} E_{\mathrm{I}_{m}}-E_{\text {graphene }}
$$

where $E_{\mathrm{I}_{n}} @$ graphene is the total energy of doped graphene with an iodine atom $(n=1)$ or a molecule $(n=2), E_{\mathrm{I}_{m}}$ is the energy of single free doping iodine atom $(m=1)$ or molecule $(m=2)$ and $E_{\text {graphene }}$ is the total energy of the pristine graphene. $E_{\mathrm{ad}}^{(1,1)}$ and $E_{\text {ad }}^{(2,2)}$ are respectively the adsorption energies of I-graphene and $\mathrm{I}_{2}$-graphene systems, while the molecular dissociation is provided by the adsorption energy $E_{\text {ad }}^{(1,2)}$.

Based on the linear dispersion of the band structure near the Dirac point, the charge carrier density (either electron or hole) of doped graphene can be estimated using the following equation: 


$$
N_{e}\left(N_{h}\right)=\frac{1}{\pi\left(\hbar v_{F}\right)^{2}}\left|E_{F}-E_{D}\right|^{2}
$$

where $N_{e}\left(N_{h}\right)$ is electron (hole) areal density, $v_{F}=1.1 \times 10^{6} \mathrm{~m} / \mathrm{s}$ is the Fermi velocity of graphene, i.e., $\hbar v_{F}=7.24 \mathrm{eV} . \AA$ extracted from our calculation on the graphene primitive cell. It is consistent with previous theoretical and experimental values. 14 133 $E_{F}$ and $E_{D}$ are the energy position of the Fermi level and of the Dirac point respectively.

We also study the effect of the temperature $T$ and the iodine activity $a$, in the socalled ab initio thermodynamics approach. It allows one to estimate the effects of the environment and the experimental conditions since the activity is a general term which can represent either the pressure $p$ in case of the perfect gas or the molarity $c$ in case of an ideal diluted solution. Experimentally, graphene layer has been in contact with a solution-deposition of $\mathrm{I}_{2} \underline{\underline{8}}$ or in iodine vapor. ${ }^{4}$ These environment acts as reservoirs for $\mathrm{I}_{2}$, giving iodine to the graphene layer without changing the temperature or the activity. The general expression of the surface free energy, $\Delta G(T, a)$ is :

$$
\Delta G(T, a)=\frac{1}{A}\left(G_{\mathrm{I}_{2} @ \text { Surf }}-G_{\text {Surf }}-\Delta N_{\mathrm{I}_{2}} \mu_{\mathrm{I}_{2}}-\Delta N_{\mathrm{C}} \mu_{\mathrm{C}}\right),
$$

where $A$ is the area of the surface unit-cell; $G_{\mathrm{I}_{2} @ \text { Surf }}$ and $G_{\text {Surf }}$ are the Gibbs free energies of the iodine-graphene system and the pristine, respectively. $\mu_{\mathrm{I}_{2}}$ and $\mu_{\mathrm{C}}$ are the chemical potentials of the iodine molecules and carbon atoms. $\Delta N_{\mathrm{I}_{2}}$ and $\Delta N_{\mathrm{C}}$ are the difference of numbers of iodine molecules and carbon atoms in the system with respect to a reference state, usually the clean surface. In this work, only the number of iodine molecules varies, while $\Delta N_{\mathrm{C}}$ is kept to zero. In general, calculations of the surface free energy of adsorption require to include variations of the vibrational energy of different structures. To overcome it, one can simply neglect all the vibrational contributions to the free Gibbs energy. ${ }^{[34}$ In this case, only the total energies are the predominant terms. The surface free energy becomes:

$$
\Delta G\left(\mu_{\mathrm{I}_{2}}\right)=\Delta E\left(\mu_{\mathrm{I}_{2}}\right) \approx \frac{1}{A}\left(E_{\mathrm{I}_{2} @ \operatorname{Surf}}-E_{\mathrm{Surf}}-\Delta N_{\mathrm{I}_{2}} \mu_{\mathrm{I}_{2}}\right)
$$


This equation establishes a direct connection between the chemical state of the surface and the reaction's conditions assuming that the surface's equilibrium state is defined as the one minimizing its energy. The other possibility is to account for the vibrational terms, but assuming that the two vibrational contributions; i.e before and after the adsorption, are equal. The surface free energy is then:

$$
\Delta G\left(\mu_{\mathrm{I}_{2}}\right) \approx \frac{1}{A}\left[E_{\mathrm{I}_{2} @ \text { Surf }}+F_{\mathrm{I}_{2} @ \text { Surf }}^{\mathrm{vib}}-E_{\mathrm{Surf}}-\Delta N_{\mathrm{I}_{2}} \mu_{\mathrm{I}_{2}}\right]
$$

where the term $F_{\mathrm{I}_{2} @ \text { Surf }}^{\mathrm{vib}}$ stands for the vibrational energy of the adsorbed species only . It can then be expressed as

$$
F_{\mathrm{I}_{2}}^{\mathrm{vib}} \mathrm{Surf}_{\mathrm{I}_{2} @ \mathrm{Sib}}^{\mathrm{vib}}-T S_{\mathrm{I}_{2} @ \text { Surf }}^{\mathrm{vib}},
$$

where $E_{\mathrm{I}_{2} @ \text { Surf }}^{\mathrm{vib}}$ and $S_{\mathrm{I}_{2} @ \text { Surf }}^{\mathrm{vib}}$ correspond to the zero-point energy and the entropic term, respectively. They are explicitly calculated using formulas extracted from. ${ }^{35}$ The $T$ and $a$ dependence is given by $\mathrm{I}_{2}$ chemical potential $\mu_{\mathrm{I}_{2}}(T, a)$ and it corresponds to

$$
\mu_{\mathrm{I}_{2}}(T, a)=E_{\mathrm{I}_{2}}+\tilde{\mu}_{\mathrm{I}_{2}}\left(T, a^{0}\right)+k_{B} T \ln \left(\frac{a_{\mathrm{I}_{2}}}{a^{0}}\right)
$$

where $E_{\mathrm{I}_{2}}$ is the energy of single free doping iodine molecule and $\tilde{\mu}_{\mathrm{I}_{2}}\left(T, a^{0}\right)$ includes rotations and vibrations of molecule. It can be either taken from first-principles calculations or deduced from experimental values (entropy and enthalpy) usually summarized in thermodynamic tables. $\frac{36}{36}$ The term $a_{\mathrm{I}_{2}} / a^{0}$ represents the ratio of the partial pressure of the $\mathrm{I}_{2}$ molecules as well as the ratio of their molarities depending on the environment.

\section{Non-local correction functionals}

In Table 1, we summarize the main geometric parameters after geometry optimizations of diatomic iodine molecule, graphene layer and graphite. They are compared with experimental data to assess the performances of few exchange-correlation functionals. LDA's value of the interatomic distance of iodine molecule is underestimated, while 
the PBE interatomic distance is in good agreement with the experimental value. Using non-local functionals, vdW-DF/DF2 and optPBE/B88-vdW tend to overestimate the I-I bond length, when optB86b-vdW's value is in good agreement with the experiment: $2.68 \AA$. All functionals give similar values, close to the experimental ones, for lattice constant and $\mathrm{C}-\mathrm{C}$ bond length, providing a good description of covalent bonds of the carbon substrate.

Concerning the interlayer distance of graphite, as usual, the LDA's value is in good agreement with the experiment $(3.33 \AA)$ but severely underestimated the binding energy, by almost $60 \%, 37$ since it does not include any long-range correlation terms in it

definition. As expected PBE underbinds the two layers with the largest value $3.99 \AA$ of the separation for all the tested functionals. When non-local corrections are added, net improvements are observed. vdW-DF and vdW-DF2 overestimate interlayer spacing by $5.8 \%(3.56 \AA)$ and $4.1 \%(3.50 \AA)$ respectively in our calculations, while optPBEvdW slightly overestimates it by $1.3 \%(3.40 \AA)$. The optB88-vdW and optB86b-vdW functionals give an interlayer distance in very good agreement with experimental value: $0.4 \%(3.34 \AA)$ and $0.7 \%(3.33 \AA)$ respectively.

Although all the tested non-local functionals are roughly equivalent, optB86b-vdW functional results are used for the following discussion.

\section{Iodine atoms on graphene layer}

We first report the variation of the adsorption energy and the height between the adatom and graphene monolayer at various concentrations. Table 2 summarizes them. Mind that there is a maximum concentration of adsorbed iodine (about 12.5\%), since beyond threshold value, adsorbed atoms get too close together rising the total energy due to a too strong repulsion between nuclei. As soon as the adatoms are enough isolated, below the $1.39 \%$ threshold value, the three adsorption sites are energetically equivalent. However above this value, the energy differences between adsorption sites remain around $20 \mathrm{meV}$ at maximum. This indicates that adatoms can surely move 
easily from one adsorption site to another, at room temperature. It can be also seen that the distance between the iodine adatom and the graphene layer is about 3.3-3.4 $\AA$, for all the concentration except the highest one. It clearly indicates that iodine adatom is physisorbed on a pristine graphene, with a relatively small value of adsorption energy.

The effects of the electron transfer between the two subsystems on the electronic structure properties of the graphene layer are shown in Fig 3 at low concentration of impurities in bridge positions, i.e 2.00\%. From the band structures in Fig 3 a, it is clear that despite the halogen atom's physisorption, , with a weak adsorption energy of -0.82 $\mathrm{eV}$, the intimate electronic structure of the graphene monolayer remains the same: the conical crossing band at $\mathrm{K}$ point is still present upon adsorption at low concentration. This is clearly different from the fluorine on graphene ${ }^{\sqrt{38}}$ case for instance. However the Dirac point is shifted above the Fermi level $(0.45 \mathrm{eV})$, corresponding to an electronic transfer from the graphene layer to the iodine atom. From Eq2, the corresponding areal hole density is around $1.2 \times 10^{13} \mathrm{~cm}^{-2}$ suggesting an effective hole doping of graphene. Besides, an impurity band due to the iodine atom appears just below the Fermi level. As shown in Fig $3 \mathrm{~b}$ this resonance, partly below the Fermi level, can be attributed to localized $p$-type orbitals of the halogen atom, with a net predominance of the $p_{x}$ state. it means that the electron of the graphene layer is transferred to occupy the last unoccupied $5 p$ state of I adatom, a clear evidence of the stronger electronegativity of the iodine atom compared to the carbon one.

Like all of the other halogens, iodine occurs naturally as a diatomic molecule $\mathrm{I}_{2}$, in solid or gaseous form. So it remains questionable to discuss the interaction of single iodine atom with graphene substrate. Energetically speaking, if one estimates the adsorption energy $E_{\text {ad }}^{(1,2)}$, which includes the dissociation energy, for each different adsorption sites and at various iodine concentrations, all the corresponding energy values are positive. For instance, for $H$ site at $5.55 \%$ and $0.50 \%$ of iodine concentration, the adsorption energies are $0.67 \mathrm{eV}$ and $0.39 \mathrm{eV}$ respectively. It thus means that the molecular state is more stable than the dissociated one. So without the presence of reactive defects on the graphene layer, the presence of single iodine adatom is highly improba- 
ble. It is therefore necessary to examine the adsorption states of non-dissociated iodine molecules on graphene layer, to obtain a more reliable atomistic picture of the doped system.

\section{Iodine molecules on graphene layer}

Table 3 summarizes the geometric and energetic properties corresponding to the adsorption of $\mathrm{I}_{2}$ molecules at various concentrations up to $12.50 \%$ since above this value the lateral interactions are too important to yield negative adsorption energy, due to a too small $\mathrm{I}_{2}-\mathrm{I}_{2}$ distances. From the values of distances between the adsorbates and the substrate, around $3.62 \AA$, as well as the adsorption energies, it is clear that the molecules are physisorbed, as already mentioned in Ref. 2. At low concentrations, i.e below $5.55 \%$, the $\mathrm{I}_{2}$ molecules prefer in-plan orientations, see Fig $2(\mathrm{a})_{\|}$, and generally gain around $0.4 \mathrm{eV}$ upon adsorption. When the center of mass of the $\mathrm{I}_{2}$ molecule is above a bridge site, the configuration is the most favorable. However energy differences between sites remain very small and certainly allow molecules to change easily from one site to the others at room temperature. When the concentration increases the adsorption mode of the di-iodine becomes perpendicular to the graphene plan. For the most dense packing, the preferred configuration is the top site, see Fig $2(i)_{\perp}$, with an adsorption energy of $-0.55 \mathrm{eV}$. One clearly observes that a modification of the iodine concentration leads to a phase transition of the adsorption mode, see Fig.4.

The density of states analysis provides useful informations about the nature of the electronic interaction between the diatomic iodine molecules and the graphene layer at different concentration. We restrict our choice of configurations to the most stable ones: at low concentrations, the in-plan orientation of $\mathrm{I}_{2},(\mathrm{a})_{\|}$, and at high concentrations, the perpendicular orientation of molecules $(i)_{\perp}$. The significant differences between density of states due to molecular concentrations are the widths and the positions of the impurity bands corresponding to the molecular states. At the highest concentration, Fig $5(\mathrm{a})$, the adsorbed iodine molecules created a new conduction band located at 
$0.8 \mathrm{eV}$ above the Fermi level. At very low concentrations, Fig 5 (c)-(d), the resonance states due to the $p$ orbitals of the halogen molecules, with a predominant $p_{x}$ character, that excludes hybridization between molecular orbitals and the $\pi$ of the graphene layer, are localized at the Fermi level. At 5.5\%, a conduction band of $0.8 \mathrm{eV}$ width appears, inducing a metallic behavior, due to overlapping of $p_{x}$ orbitals from different $\mathrm{I}_{2}$ molecules, since they are only separated by $4.67 \AA$, in agreement with a previous study. $[2$

Considering the electronic transfer from the substrate to the molecules, at low concentration, a small shift of the Dirac point is observed, for instance the shift is $0.08 \mathrm{eV}$ at $2 \%$ of iodine concentration. The corresponding areal hole density is around $3.9 \times 10^{11}$ $\mathrm{cm}^{-2}$. In Table 3 the effective charges of the adsorbed $\mathrm{I}_{2}$ molecules are also given. The total amount of charge transferred is much weaker for molecules than for single atoms. If the molecules have in-plan orientation, i.e at low concentration, atomic charges are equivalent. However they are different for perpendicular adsorption mode, the closer atom to the graphene layer taking more electronic density, in ratio $2 / 3-1 / 3$. Figure 6 , presents both the graphene effective charge and the corresponding hole areal density, per unit cell, for the two most stable orientations with respect to the iodine coverage values. The iodine concentration of $12.50 \%$ is taken as reference for the maximum coverage value, i.e. $100 \%$. The hole areal density is maximal, $1.1 \times 10^{13} \mathrm{~cm}^{-2}$, when the adsorbed iodines are in-plan with a coverage value of $88 \%$. In other words, this value corresponds to a Raman shift of approximately $9 \mathrm{~cm}^{-1}$ using the procedure proposed in

Ref. 14. This value agrees well with a recent experimental estimate. ${ }^{3}$ In conclusion the conductivity of graphene can be adjusted by changing the concentration of $\mathrm{I}_{2}$, which depends crucially on the molecular orientations. The thermodynamics stability of these phases are studied in the following part.

\section{$(T, a)$ phase-diagram}

The variation of the Gibbs free energies, including vibrational corrections, of stable structures at different coverage values, as a function of the $\mathrm{I}_{2}$ chemical potential are 
given in Fig. 7. Since in experimental settings, $\frac{8}{8}$ iodine can be considered as the majority species, the ratio $a_{\mathrm{I}_{2}} / a^{0}$ is equal to 1 . This diagram has three distinct zones representing the most stable adsorption states as a function of the temperature. In zone 1 (blue), molecules are absorbed perpendicularly with a dense packing on the substrate. Beyond $T_{1}=219 \mathrm{~K}$, occurs a phase transition, zone 2 (red), where the supply of thermal energy causes the desorption of some molecules, allowing others to have inplan orientation. The molecules are still adsorbed below $T_{2}=511 \mathrm{~K}$. Beyond, zone 3 (green), the graphene layer is clean. This is in good agreement, with the experimental observations of Ref 8 , in which the desorption is obtained at $T_{2-\exp }=573 \mathrm{~K}$.

\section{Conclusion}

In this paper, the study of the adsorption of iodine atoms and molecules on graphene using DFT calculations, including non-local correlation correction, are reported, including the energetic and geometric aspects, as well as the electronic properties of the doped systems. As already reported, single iodine adatoms are physisorbed and withdraw a consequent amount of charge from the undeformed graphene sheet. The iodine atom accepts 0.46 electron and the corresponding hole areal density is around $1.2 \times 10^{13}$ $\mathrm{cm}^{-2}$. However the molecular state is more stable than the dissociated one, It is therefore mandatory to examine the adsorption states of non-dissociated iodine molecules on graphene layer. Interestingly, the adsorption of $\mathrm{I}_{2}$ molecules exhibits a phase transition from in-plan to perpendicular orientation when concentration increases with a maximum value of $12.50 \%$. Besides iodine molecules can easily migrate from one adsorption site to another. At low concentration, impurity bands in the vicinity of the Fermi level appear, and at the concentration of $5.5 \%$ a new conduction channel is opened. It turns out that a effective charge transfer of $5.55 \times 10^{-3}$ electron per graphene unit cell to the $\mathrm{I}_{2}$ molecule is yielded, and corresponds to a hole areal density of around $1.1 \times 10^{13}$

$\mathrm{cm}^{-2}$. This provides in Raman spectra an upshift of $9 \mathrm{~cm}^{-1}$ in the G-band, that could be modulated by the $\mathrm{I}_{2}$ concentration. Additionally we have studied the effects of 
temperature and of the activity of the iodine reservoir, to the adsorption modes, with a desorption temperature beyond $500 \mathrm{~K}$. The present work could serve as a basis for further experimental and theoretical studies on molecular adsorption on graphene. But more importantly it will serve as a first step towards a better understanding of the doping mechanism of carbon based nanostructures by iodine.

\section{Acknowledgments}

D. Tristant thanks the Midi-Pyrénées Région and the PRES Université de Toulouse for PhD funding. The authors also acknowledge the Calcul en Midi-Pyrénées initiativeCALMIP (Project p0812) for allocations of computer time. Part of this work was also performed using HPC resources from GENCI-IDRIS (Project x2014096649). VESTA software is gratefully acknowledged for atomic representations. We also thank Romuald Poteau for fruitful discussions. 


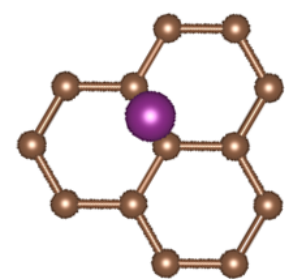

(a)

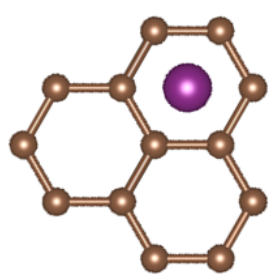

(b)

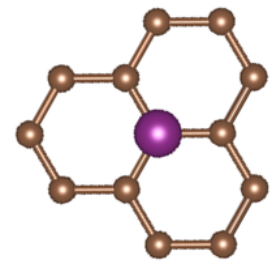

(c)

Figure 1: (Color online) Top view of different high-symmetry adsorption sites for iodine atom (purple circle) on the graphene layer (brown circles): (a) bridge $B$, (b) hollow $H$ and (c) top $T$ sites. 


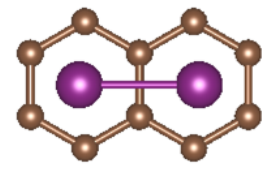

(a)

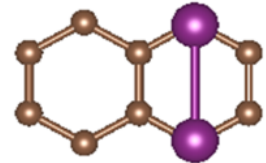

(d)

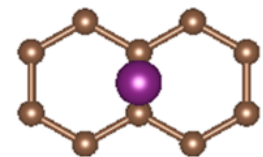

$(\mathrm{g})_{\perp}$

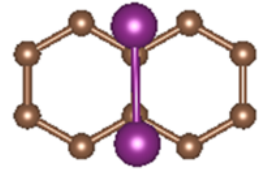

(b)

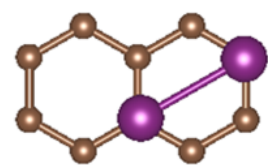

(e)

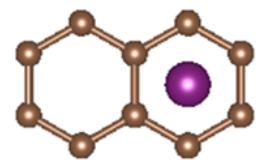

(h)

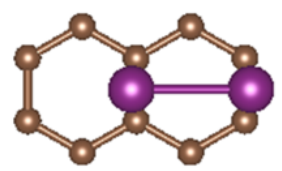

(c)

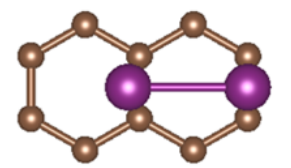

(f)

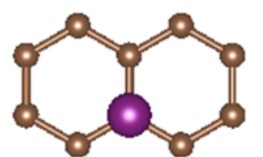

$(\mathrm{i})_{\perp}$

Figure 2: (Color online) Top view of different high-symmetry adsorption sites of a diatomic iodine molecule (purple circles) on a graphene layer (brown circles). The first six configurations have an in-plan orientation of the molecule, the center of mass is located above: (a)(b) a bridge site along $x$ and $y$ axis respectively, (c)(d)(e) a hollow site along $x, y$ and diagonal axis respectively and (f) one of both atom is in the bridge site while the other is not localized in high-symmetry adsorption site. The last three configurations corresponds to a perpendicular orientation of a molecule on top of : (g) a bridge site, (h) a hollow site and (i) a top site. 
Table 1: Interatomic distance of iodine molecule $d_{\mathrm{I}-\mathrm{I}}(\AA)$, lattice constant $a_{0}(\AA)$ and carboncarbon bond lenghts $a_{\mathrm{C}-\mathrm{C}}(\AA)$ of graphene layer and interlayer distance of graphite (ABstacked layers) $c_{0}(\AA)$ obtained in this work. They are compared with experimental and other calculated values using various exchange-correlation functionals.

\begin{tabular}{|c|c|c|c|c|c|c|c|c|}
\hline & \multicolumn{2}{|c|}{ local \& semi-local } & \multicolumn{5}{|c|}{ non-local vdW } & \multirow[b]{2}{*}{ exp. } \\
\hline & LDA & PBE & $-\mathrm{DF}$ & -DF2 & optPBE & optB88 & optB86b & \\
\hline$d_{\mathrm{I}-\mathrm{I}}$ & 2.64 & 2.68 & 2.72 & 2.76 & 2.70 & 2.69 & 2.68 & $2.666^{18}$ \\
\hline$a_{0}$ & 2.46 & 2.46 & 2.46 & 2.46 & 2.46 & 2.46 & 2.46 & $2.459^{17}$ \\
\hline$a_{\mathrm{C}-\mathrm{C}}$ & $\begin{array}{c}1.42^{37} \\
1.412^{37} \\
1.413^{40}\end{array}$ & $\begin{array}{c}1.42 \\
1.424^{37}\end{array}$ & 1.42 & $\begin{array}{c}1.42 \\
1.428^{37}\end{array}$ & $\begin{array}{c}1.42 \\
1.426^{37}\end{array}$ & $\begin{array}{c}1.42 \\
1.422^{37}\end{array}$ & 1.42 & $1.421^{39}$ \\
\hline$c_{0}$ & $\begin{array}{c}3.33 \\
3.31^{137} \\
3.29^{40} \\
3.30^{43} \\
3.33^{45}\end{array}$ & $\begin{array}{c}3.99 \\
\sim 43\end{array}$ & $\begin{array}{c}3.56 \\
3.5941\end{array}$ & $\begin{array}{c}3.50 \\
3.54^{\sqrt{37}} \\
3.47^{42} \\
3.48^{44}\end{array}$ & $\begin{array}{c}3.40 \\
3.46^{\text {37 }}\end{array}$ & $\begin{array}{c}3.34 \\
3.36^{37}\end{array}$ & 3.33 & $3.354^{19}$ \\
\hline
\end{tabular}


Table 2: Variation of the adsorption energy $E_{\text {ad }}(\mathrm{eV})$ and the adsorption height between the adatom and the average height of the graphene layer $h_{\text {I-gra }}(\AA)$, for different iodine adatom concentrations and at different high-symmetry adsorption sites: the hollow $(H)$, the top $(T)$ and the bridge $(B)$ sites. When available values from the literature, at the PBE level are reported.

\begin{tabular}{|c|c|c|c|c|c|c|}
\hline \multirow{2}{*}{$\begin{array}{c}\text { Iodine } \\
\text { concentration }(\%)\end{array}$} & \multicolumn{2}{|c|}{$H$ site } & \multicolumn{2}{|c|}{$T$ site } & \multicolumn{2}{|c|}{$B$ site } \\
\hline & $E_{\mathrm{ad}}^{(1,1)}$ & $h_{\text {I-gra }}$ & $E_{\mathrm{ad}}^{(1,1)}$ & $h_{\text {I-gra }}$ & $E_{\mathrm{ad}}^{(1,1)}$ & $h_{\text {I-gra }}$ \\
\hline 12.50 & -0.76 & 3.52 & -0.76 & 3.57 & -0.76 & 3.56 \\
\hline 5.55 & -0.65 & 3.41 & -0.66 & 3.32 & $\begin{array}{c}-0.65 \\
-0.34^{5}\end{array}$ & 3.34 \\
\hline 3.12 & -0.74 & 3.41 & -0.75 & 3.33 & $\begin{array}{c}-0.76 \\
-0.44^{5}\end{array}$ & 3.33 \\
\hline 2.00 & -0.81 & 3.41 & -0.82 & 3.36 & $\begin{array}{c}-0.82 \\
-0.51^{5}\end{array}$ & 3.36 \\
\hline 1.39 & $\begin{array}{l}-0.86 \\
-0.51^{5}\end{array}$ & 3.37 & $\begin{array}{l}-0.86 \\
-0.52^{5}\end{array}$ & 3.35 & $\begin{array}{l}-0.86 \\
-0.54^{5}\end{array}$ & $\begin{array}{c}3.33 \\
3.77^{5}\end{array}$ \\
\hline 1.02 & -0.89 & 3.39 & -0.89 & 3.35 & -0.89 & 3.35 \\
\hline 0.78 & -0.91 & 3.39 & -0.91 & 3.35 & -0.91 & 3.36 \\
\hline 0.62 & -0.92 & 3.40 & -0.92 & 3.36 & -0.92 & 3.35 \\
\hline 0.50 & -0.93 & 3.40 & -0.93 & 3.37 & -0.93 & 3.36 \\
\hline
\end{tabular}




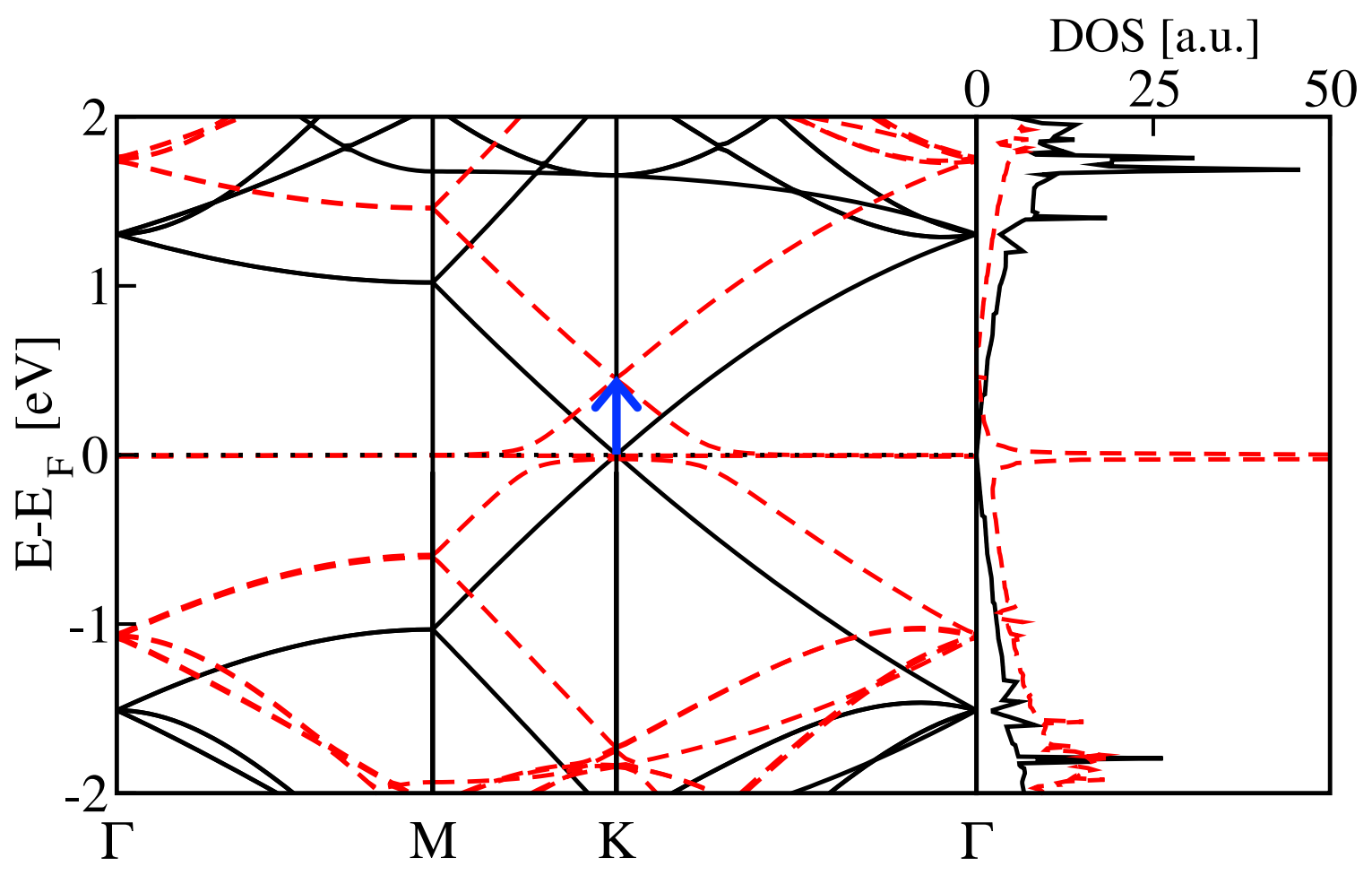

Figure 3: (Color online) Band structure (a) and total electronic density of states (b) of isolated graphene layer (black line) and with halogen atoms adsorbed on graphene layer (red dash line) at low iodine concentration (2.00\%). The blue arrow shows the shift of Dirac point. The Fermi level is at zero energy. 
Table 3: Variation of the adsorption energy $E_{a d}^{(2,2)}(\mathrm{eV})$, of the effective charge of $\mathrm{I}_{2}$ (number of electron), of the adsorption height between the molecules and the average height of the graphene layer $(\AA)$, of the minimum distance between two $I_{2}(\AA)$ and the bond distance between the two iodine atoms $(\AA)$, for various coverage values in different high-symmetry adsorption sites.

\begin{tabular}{|c|c|c|c|c|c|c|}
\hline $\begin{array}{l}\text { Adsorption } \\
\text { site }\end{array}$ & $\begin{array}{c}\text { Iodine } \\
\text { concentration }(\%)\end{array}$ & $\begin{array}{l}\text { Adsorption } \\
\text { energy }\end{array}$ & $\begin{array}{c}\mathrm{I}_{2} \text {-graphene } \\
\text { distance }\end{array}$ & $\begin{array}{c}\mathrm{I}_{2}-\mathrm{I}_{2} \\
\text { distance }\end{array}$ & $\begin{array}{l}\text { I-I bond } \\
\text { length }\end{array}$ & $\begin{array}{c}\mathrm{I}_{2} \text { Effective } \\
\text { charge }\end{array}$ \\
\hline \multirow{6}{*}{$(a)_{\|}$} & 5.55 & -0.48 & 3.67 & 4.67 & 2.71 & -0.05 \\
\hline & 4.16 & $-0.523^{2}$ & $3.80^{2}$ & $\sim 6.47$ & & \\
\hline & 3.12 & -0.41 & 3.63 & 7.13 & 2.71 & -0.03 \\
\hline & 2.00 & -0.42 & 3.66 & 9.59 & 2.71 & -0.03 \\
\hline & 1.39 & -0.42 & 3.59 & 12.05 & 2.71 & -0.03 \\
\hline & 1.02 & -0.42 & 3.53 & 14.51 & 2.71 & -0.03 \\
\hline \multirow{2}{*}{$(b)_{\|}$} & 4.16 & $-0.512^{2}$ & $3.81^{2}$ & $\sim 5.32$ & & \\
\hline & 2.00 & -0.42 & 3.63 & 12,30 & 2.71 & -0.02 \\
\hline \multirow{2}{*}{$(\mathrm{c})_{\|}$} & 4.16 & $-0.514^{2}$ & $3.84^{2}$ & $\sim 6.47$ & & \\
\hline & 2.00 & -0.40 & 3.68 & 9.59 & 2.71 & -0.02 \\
\hline \multirow{2}{*}{$(d)_{\|}$} & 4.16 & $-0.508^{2}$ & $3.83^{2}$ & $\sim 5,32$ & & \\
\hline & 2.00 & -0.41 & 3.64 & 12.30 & 2.71 & -0.02 \\
\hline \multirow{2}{*}{$(\mathrm{e})_{\|}$} & 4.16 & $-0.501^{2}$ & $3.83^{2}$ & $\sim 5,21$ & & \\
\hline & 2.00 & -0.41 & 3.65 & 11.08 & 2.71 & -0.03 \\
\hline$(\mathrm{f})_{\|}$ & 2.00 & -0.41 & 3.65 & 9.59 & 2.71 & -0.02 \\
\hline \multirow{5}{*}{$(\mathrm{g})_{\perp}$} & 12.50 & -0.54 & 3.24 & 4.91 & 2.69 & -0.02 \\
\hline & 5.55 & -0.38 & 3.18 & 7.38 & 2.70 & -0.03 \\
\hline & 4.16 & $-0.375^{2}$ & $4.74^{2}$ & $\sim 7,38$ & & \\
\hline & 3.12 & -0.36 & 3.15 & 9.84 & 2.70 & -0.04 \\
\hline & 2.00 & -0.36 & 3.52 & 12.30 & 2.70 & -0.04 \\
\hline \multirow{5}{*}{$(\mathrm{h})_{\perp}$} & 12.50 & -0.50 & 3.39 & 4.91 & 2.69 & 0.00 \\
\hline & 5.55 & -0.33 & 3.33 & 7.38 & 2.69 & -0.01 \\
\hline & 4.16 & $-0.352^{2}$ & $4.85^{2}$ & $\sim 7,38$ & & \\
\hline & 3.12 & -0.31 & 3.29 & 9.84 & 2.69 & -0.01 \\
\hline & 2.00 & -0.31 & 3.26 & 12.30 & 2.69 & -0.01 \\
\hline \multirow{5}{*}{$(\mathrm{i})_{\perp}$} & 12.50 & -0.55 & 3.22 & 4.91 & 2.69 & -0.01 \\
\hline & 5.55 & -0.38 & 3.17 & 7.38 & 2.70 & -0.03 \\
\hline & 4.16 & $-0.379^{2}$ & $4.73^{2}$ & $\sim 7,38$ & & \\
\hline & 3.12 & -0.37 & 3.14 & 9.84 & 2.70 & -0.04 \\
\hline & 2.00 & -0.37 & 3.12 & 12.30 & 2.71 & -0.05 \\
\hline
\end{tabular}




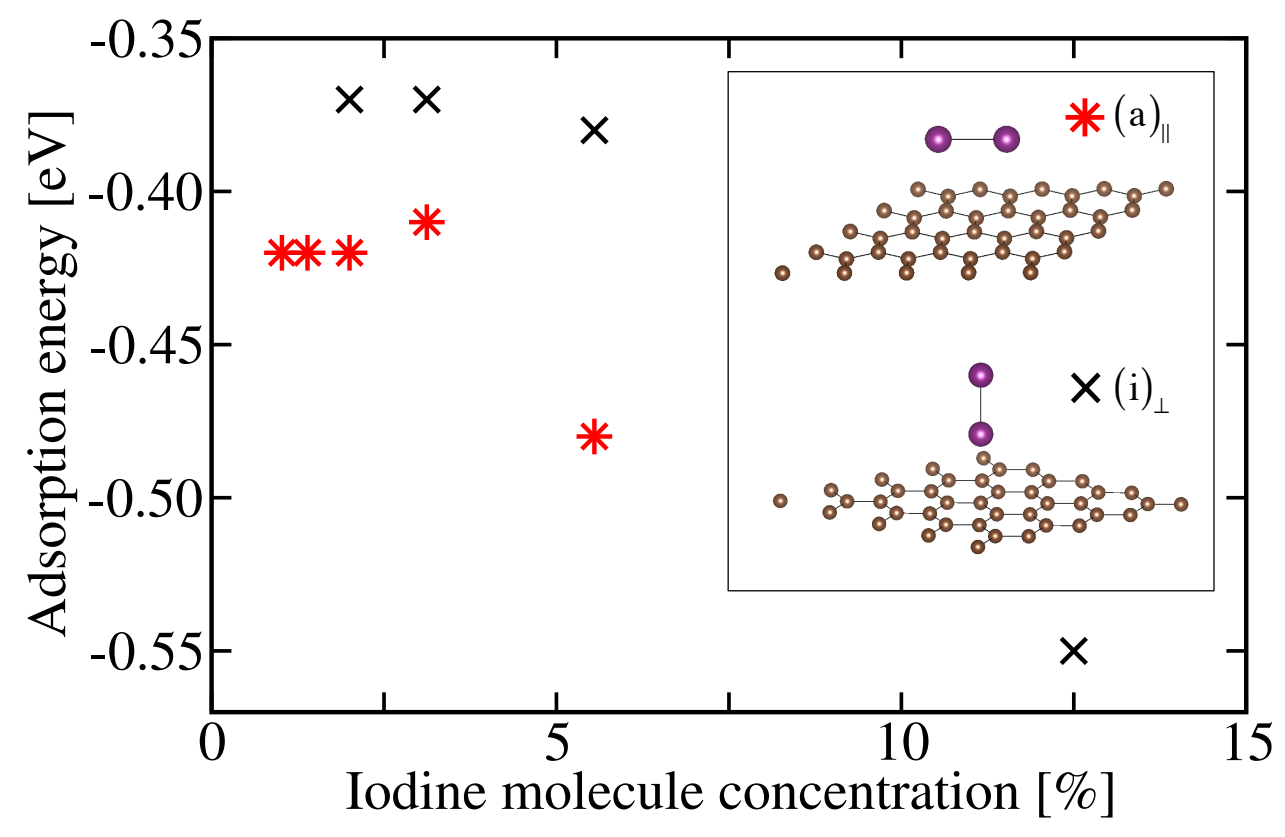

Figure 4: (Color online) Variation of the adsorption energy $(\mathrm{eV})$ at different iodine molecule concentrations in several high-symmetry adsorption sites: the bridge site along $x$ (a) $\|$ (red star) and the top site of hexagonal cell (i $)_{\perp}$ (black cross). 


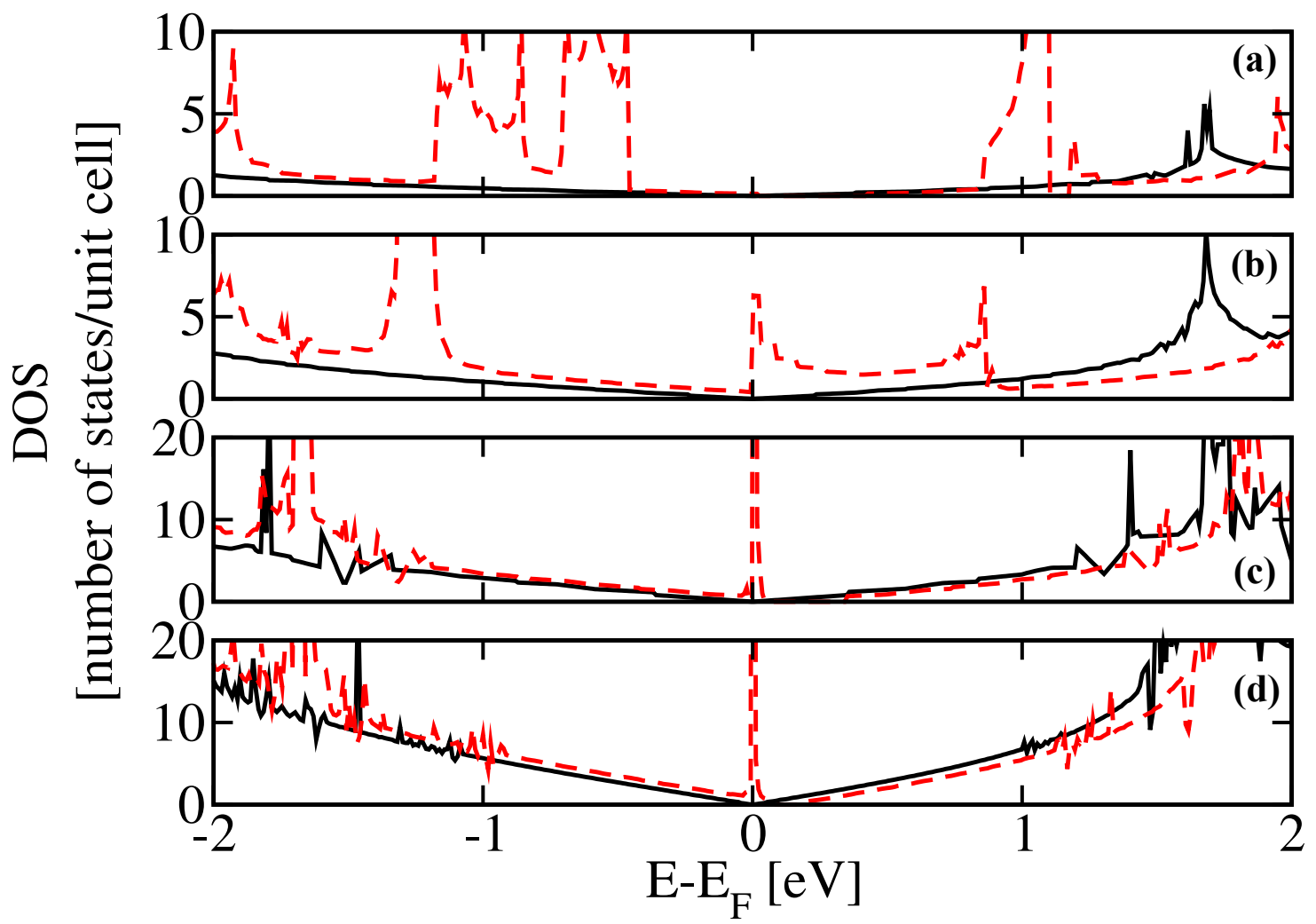

Figure 5: (Color online) Total density of states of the isolated graphene layer (black line) and diatomic iodine molecules adsorbed on graphene layer (red dash line) for various adsorbate concentrations: (a) $12.5 \% \perp$; (b) $5.55 \% \|$; (c) $2.00 \% \|$ and (d) $1.02 \% \|$. The Fermi level is set to be zero energy. 


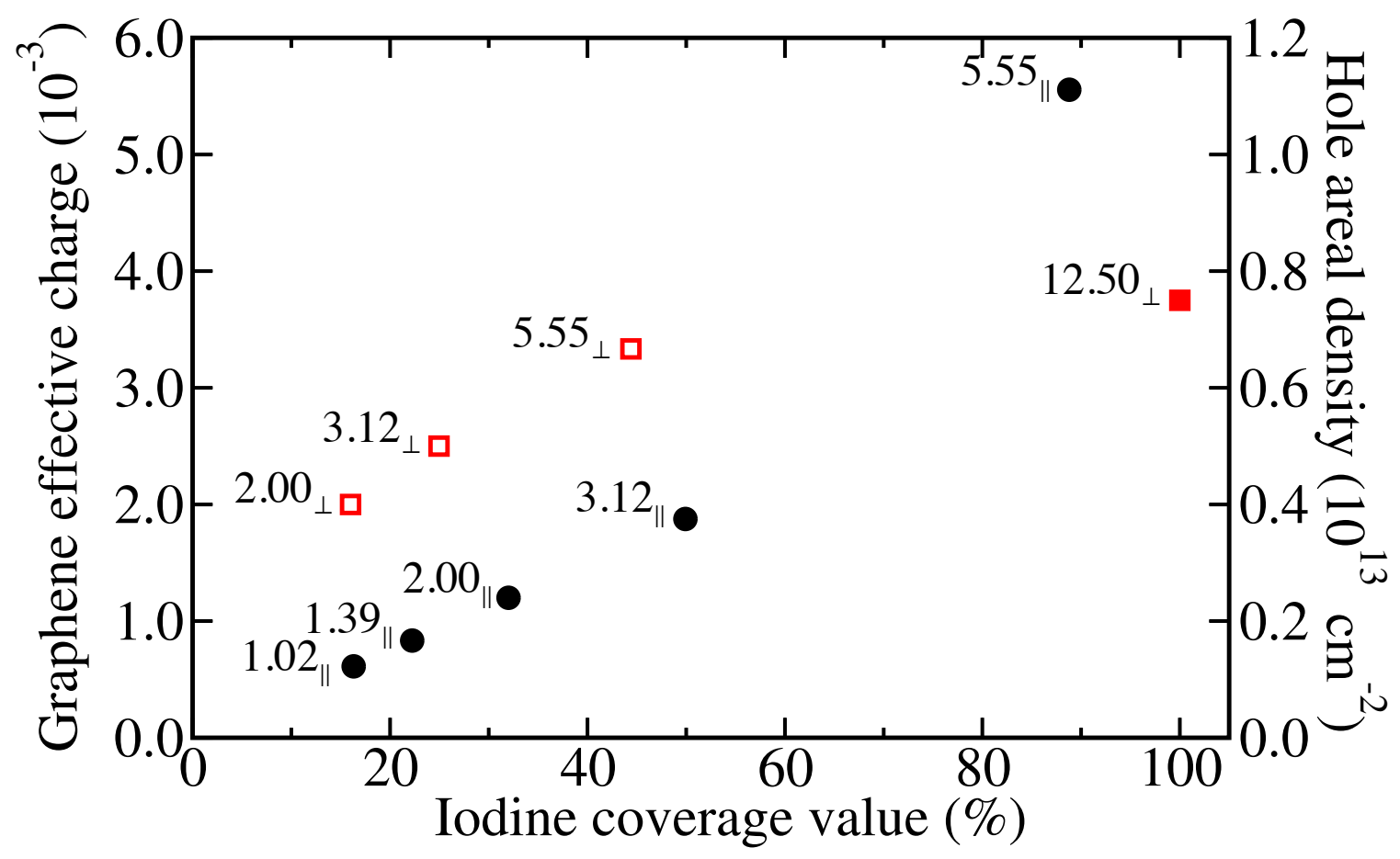

Figure 6: (Color online) Graphene effective charge and hole areal density per unit cell as a function of the iodine coverage value. Each point is annotated with its iodine concentration, and corresponds to the following adsorbed iodine orientation: (o) in-plan and ( $\square$ ) perpendicular. Full dots $(\bullet$ and $)$ represent the most stable adsorption states. 


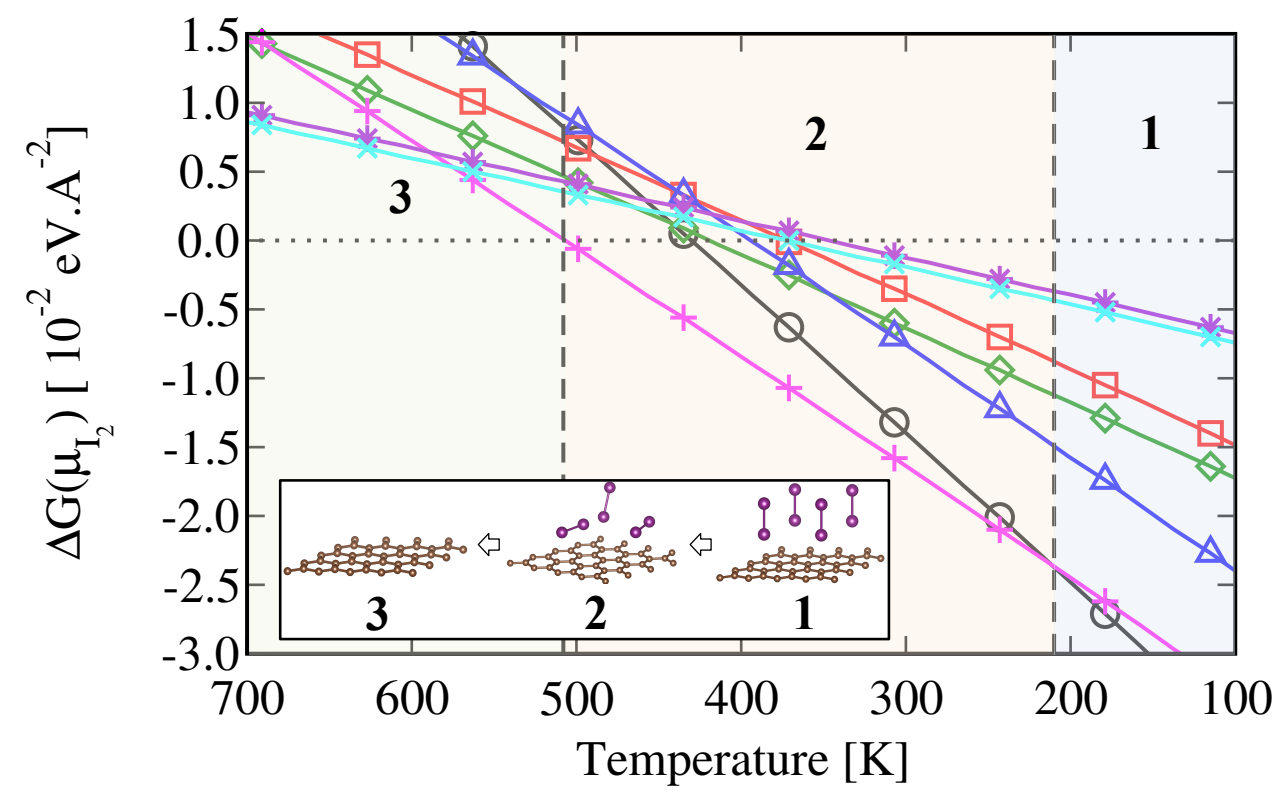

Figure 7: (Color online) Variations of Gibbs free energy including vibrational contributions as a function of the $\mathrm{I}_{2}$ chemical potential at different coverage. Each line corresponds to the following iodine concentration value: $(\bigcirc)=12.5 \%(\mathrm{i})_{\perp} ;(+)=6.25 \%(\mathrm{i})_{\perp}+3.13 \%(\mathrm{a})_{\|}$; $(\triangle)=9.38 \%(\mathrm{i})_{\perp} ;(\diamond)=6.25 \%(\mathrm{a})_{\|} ;(\square)=6.25 \%(\mathrm{i})_{\perp} ;(\times)=3.13 \%(\mathrm{a})_{\|} ;(*)=3.13 \%(\mathrm{i})_{\perp}$. The iodine chemical potential has been converted to the corresponding temperatures at standard $\left(a_{\mathrm{I}_{2}} / a^{0}=1\right) \mathrm{I}_{2}$ activity. 


\section{Bibliography}

(1) Vala, J.; Kosloff, R.; Harvey, J. N. The Journal of Chemical Physics 2001, 114, $7413-7423$.

(2) Rudenko, A. N.; Keil, F. J.; Katsnelson, M. I.; Lichtenstein, A. I. Phys. Rev. B 2010, 82, 035427.

(3) Chu, S. W.; Baek, S. J.; Kim, D. C.; Seo, S.; Kim, J. S.; Park, Y. W. Synthetic Metals 2012, 162, 1689-1693.

(4) Ghosh, S.; Yamijala, S. S.; Pati, S. K.; Rao, C. RSC Advances 2012, 2, 1181-1188.

(5) Luo, X.; Fang, C.; Li, X.; Lai, W.; Liang, T. Journal of Nuclear Materials 2013, $441,113-118$.

(6) Davydov, S. Y.; Sabirova, G. Technical Physics Letters 2011, 37, 515-518.

(7) Wang, Z.; Wang, W.; Wang, M.; Meng, X.; Li, J. Journal of Materials Science 2013, 48, 2284-2289.

(8) D'Arsié, L.; Esconjauregui, S.; Weatherup, R.; Guo, Y.; Bhardwaj, S.; Centeno, A.; Zurutuza, A.; Cepek, C.; Robertson, J. Applied Physics Letters 2014, 105, 103103.

(9) Fan, X.; Liu, L.; Kuo, J.-L.; Shen, Z. The Journal of Physical Chemistry C 2010, $114,14939-14945$.

(10) Ijäs, M.; Havu, P.; Harju, A. Phys. Rev. B 2012, 85, 035440.

(11) Dion, M.; Rydberg, H.; Schröder, E.; Langreth, D. C.; Lundqvist, B. I. Phys. Rev. Lett. 2004, 92, 246401.

(12) Chen, Z.; Darancet, P.; Wang, L.; Crowther, A. C.; Gao, Y.; Dean, C. R.; Taniguchi, T.; Watanabe, K.; Hone, J.; Marianetti, C. A.; Brus, L. E. ACS Nano 2014, 8, 2943-2950. 
(13) Puech, P.; Hu, T.; Sapelkin, A.; Gerber, I.; Tishkova, V.; Pavlenko, E.; Levine, B.; Flahaut, E.; Bacsa, W. Phys. Rev. B 2012, 85, 205412.

(14) Lazzeri, M.; Mauri, F. Phys. Rev. Lett. 2006, 97, 266407.

(15) Zhao, Y.; Wei, J.; Vajtai, R.; Ajayan, P. M.; Barrera, E. V. Scientific Reports 2011,1 .

(16) Klimeš, J.; Bowler, D. R.; Michaelides, A. Phys. Rev. B 2011, 83, 195131.

(17) Baskin, Y.; Meyer, L. Phys. Rev. 1955, 100, 544-544.

(18) Pauling, L. The Nature of the Chemical Bond and the Structure of Molecules and Crystals: An Introduction to Modern Structural Chemistry; $3^{\text {rd }}$ ed. Cornell University Press, Ithaca, New York, 1960.

(19) Lewin, M. Handbook of Fiber Chemistry; $3^{\text {rd }}$ ed. International Fiber Science and Technology, CRC Press, p. 944, 2006.

(20) Kresse, G.; Hafner, J. Phys. Rev. B 1993, 47, 558-561.

(21) Kresse, G.; Hafner, J. Phys. Rev. B 1994, 49, 14251-14269.

(22) Kresse, G.; Furthmüller, J. Phys. Rev. B 1996, 54, 11169-11186.

(23) Kresse, G.; Furthmüller, J. Computational Materials Science 1996, 6, 15 - 50.

(24) Blöchl, P. E. Physical Review B 1994, 50, 17953.

(25) Fu, C. L.; Ho, K. M. Phys. Rev. B 1983, 28, 5480-5486.

(26) Zhang, Y. K.; Yang, W. T. Phys. Rev. Lett. 1998, 80, 890-890.

(27) Klimeš, J. c. v.; Bowler, D. R.; Michaelides, A. Phys. Rev. B 2011, 83, 195131.

(28) Perdew, J. P.; Burke, K.; Ernzerhof, M. Phys. Rev. Lett. 1996, 77, 3865-3868.

(29) Blöchl, P. E.; Jepsen, O.; Andersen, O. K. Phys. Rev. B 1994, 49, 16223-16233. 
(30) Tang, W.; Sanville, E.; Henkelman, G. Journal of Physics: Condensed Matter 2009, 21, 084204.

(31) Sanville, E.; Kenny, S. D.; Smith, R.; Henkelman, G. Journal of computational chemistry 2007, 28, 899-908.

(32) Henkelman, G.; Arnaldsson, A.; Jónsson, H. Computational Materials Science 2006, 36, 354-360.

(33) Li, G.; Luican, A.; Andrei, E. Y. Phys. Rev. Lett. 2009, 102, 176804.

(34) Reuter, K.; Scheffler, M. Phys. Rev. B 2001, 65, 035406.

(35) McQuarrie, D. Statistical Mechanics, 2nd ed.; University Science Books: Sausalito, California, 2000.

(36) Stull, D.; Prophet, H. JANAF Thermochemical Tables, 2nd ed.; 1971.

(37) Graziano, G.; Klimeš, J.; Fernandez-Alonso, F.; Michaelides, A. Journal of Physics: Condensed Matter 2012, 24, 424216.

(38) Karlicky, F.; Kumara Ramanatha Datta, K.; Otyepka, M.; Zboril, R. ACS nano 2013, 7, 6434-6464.

(39) Saito, R.; Dresselhaus, M. S.; Desselhaus, G. Physical Properties of Carbon Nanotubes; Imperial College Press, 1998.

(40) Rairkar, A.; Adams, J. B.; Ooi, N. Carbon 2006, 44, 231.

(41) Ziambaras, E.; Kleis, J.; Schröder, E.; Hyldgaard, P. Phys. Rev. B 2007, 76, 155425.

(42) Hamada, I.; Otani, M. Phys. Rev. B 2010, 82, 153412.

(43) Spanu, L.; Sorella, S.; Galli, G. Phys. Rev. Lett. 2009, 103, 196401. 
(44) Berland, K.; Borck, O.; Hyldgaard, P. Computer Physics Communications 2011, 182, 1800.

(45) Lebègue, S.; Harl, J.; Gould, T.; Ángyán, J. G.; Kresse, G.; Dobson, J. F. Phys. Rev. Lett. 2010, 105, 196401. 
Table of content graphical abstract:

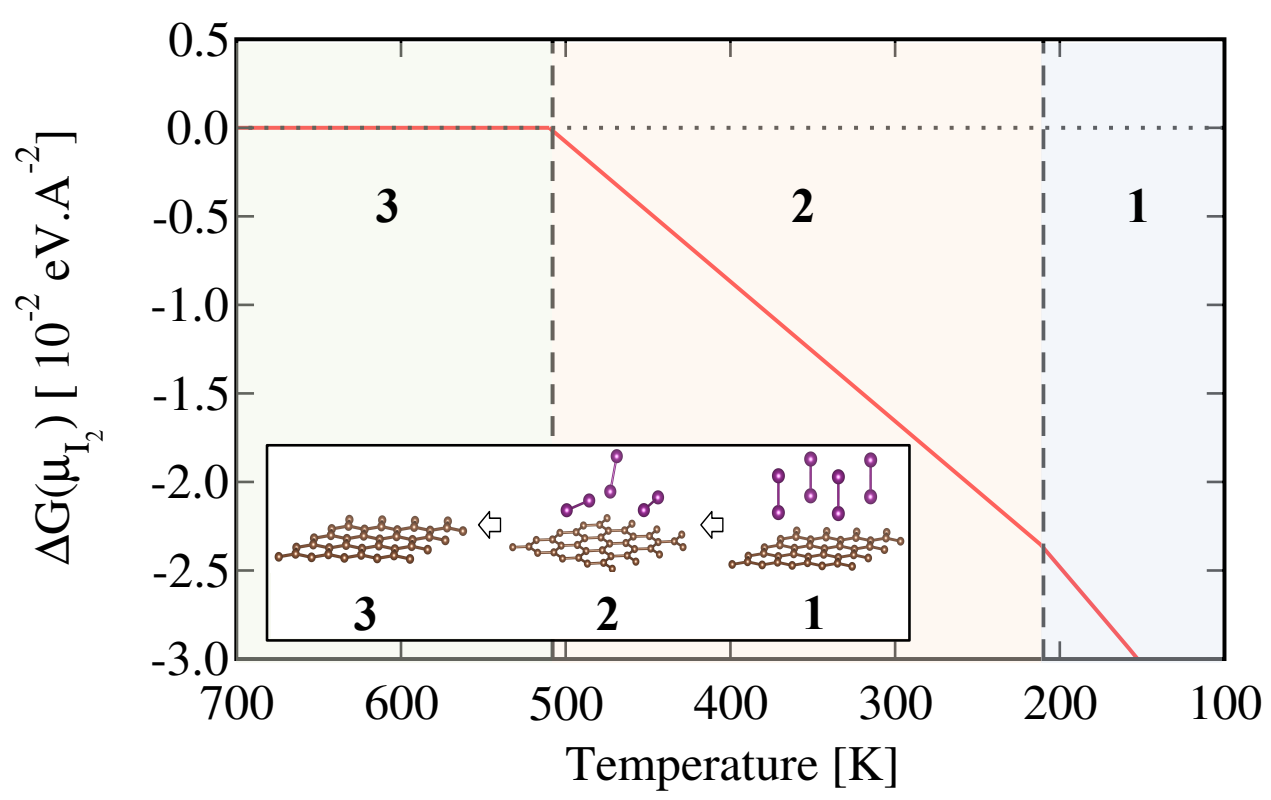

